\title{
Interface Controlled Multiple Elimination by Sparsity Inversion
}

\author{
Lanshu Bai ${ }^{1, *}$, Yike $\mathrm{Liu}^{2}$ and Huiyi $\mathrm{Lu}^{3}$ \\ ${ }^{1}$ Institute of Geophysics, China Earthquake Administration, Beijing 100081, China. \\ ${ }^{2}$ Institute of Geology and Geophysics, Chinese Academy of Sciences, Beijing 100029, \\ China. \\ ${ }^{3}$ Kerogen Energy Services Co., Ltd, Beijing 100101, China.
}

Received 27 March 2018; Accepted (in revised version) 22 February 2019

\begin{abstract}
Removing internal multiples remains an important but challenging problem in seismic processing. The generalized Estimation of Primaries by Sparsity Inversion (EPSI) method minimizes data residuals between the calculated and observed waveform using the sparse constraint of primary impulse responses to predict multiples and remove them directly, instead of using the conventional adaptive subtraction method. Even though the generalized EPSI method provides a good estimate of the primaries and multiples when they overlap, it is limited by intensive computational cost.

In this paper, we introduce two strategies to improve computational efficiency. First, the interface-controlled strategy is introduced by only selecting high-amplitude primary responses related to the interfaces with strong impedance contrasts to estimate multiples. The computational time is approximately proportional to the number of involved reflectors and usually, most of the internal multiple energy in the data is only related to a few strong reflectors. Therefore the modified method can remove most of the internal multiples in fewer computations than in the generalized EPSI, which loops through all the interfaces. Next, an approximate formula for estimating primary impulse responses is proposed by neglecting a computationally intensive term which corresponds to the primary responses estimated from internal multiples. According to our analyses and experiments, in most cases, the contribution of this term is negligible because the internal multiples are weak. Therefore, the computational efficiency can be improved without significantly losing quality when estimating most primaries and multiples.

In order to demonstrate this, multiple elimination of a two-layered simple data and the Pluto data are implemented. We find that the modified method can yield reliable results that require fewer computations. The improvements of the modified method may encourage the use of the generalized EPSI method in industry.
\end{abstract}

AMS subject classifications: $86-08,86 \mathrm{~A} 15,86 \mathrm{~A} 22$

*Corresponding author. Email addresses: bailanshu@cea-igp.ac.cn (L. Bai), ykliu@mail.iggcas.ac.cn (Y. Liu), Ihy061981@163.com (H. Lu) 
Key words: Multiple, internal multiple, interface-controlled multiple elimination, estimation of primaries by sparsity inversion.

\section{Introduction}

Primary reflections are dominating in seismic datasets and considered as the most effective data for most popular subsurface imaging techniques in the surface reflection seismic exploration. Therefore, multiples especially in marine data are regarded as noises reducing imaging quality in most processing algorithms. The multiples (including internal and surface-related multiples) significantly contaminate subsurface images and thus reduce the reliability of reservoir prediction and well location design. In shallow water data, the reverberation caused by multiple reflections between free surface and the seabed is such a serious problem that it is very tough to distinguish the primaries without suppressing multiples. In recent years, multiple subsurface imaging techniques and some primary and multiple joint imaging techniques, that exploit multiples instead of removing them, have been developed to improve the illumination in subsalt area [1-5]. However, in most applications, especially in oil and gas industry, the primaries and multiples are still required to be separated before the seismic imaging. Therefore, accurate estimation of primaries and multiples is still an important aspect of both primary imaging techniques and the multiple imaging techniques.

Multiples are defined as waves that have undergone more than one upward reflection before they are measured (ghost waves are not included). The upward reflection indicates the upgoing waves that reflect at the interface and turn to propagate upward. According to the position that the upgoing reflection takes place during the propagation, multiples can be divided into two types: the surface-related multiples and the internal multiples. Surface-related multiples are multiples that reflect at the surface at least once, and the internal multiples are those that reverberate in the subsurface interfaces without surface reflections. For instance, in marine exploration seismology, surface-related multiples are multiples that reflect at the sea surface, and internal multiples are those that reflect at the sea bottom, sediment interfaces and other impedance interfaces, such as salt dome boundaries.

Over the past few decades, two types of methods for suppressing multiples have been developed and optimized: the filtering method and the wave-theory-based method. Filtering techniques are based on signal analysis, which includes domain transform filter and predictive deconvolution [6-8]. These methods have been widely used and have been very successful in industry. However, it is difficult to estimate non-periodic multiples produced by the complex underground structures. Wave equation-based methods make full use of the kinematic and dynamic characteristics of wave propagation to estimate the primaries and multiples. Wave equation-based methods can be further divided into two categories: the two-step prediction-subtraction method and the sparse constraint inversion method. In the prediction-subtraction multiple elimination approach, 
the multiples are first predicted by data-driven or model-driven methods, and then the multiples are eliminated by adaptive subtraction. These methods include Surface-Related Multiple Elimination (SRME) [9-12], Inverse Scattering Series (ISS) [13], Internal Multiple Elimination (IME) [14-16] and other model-driven methods [17-20]. Keydar et al. [21] proposed that first-order internal multiples can be predicted based on a "low-high-low" reflection mode by a multidimensional convolution and correlation of three primaries, and then subtracted from the original data. The IME approach [14-16] is an extension of the SRME method for estimating of internal multiples and its use has been demonstrated in field data applications [22-24]. Theoretically, the ISS method can estimate the internal multiples related to all the reflectors, but in practice, it is limited by the large computational demands and poor practicality. Berkhout and Verschuur used Common Focus Point (CFP) data gathers to estimate the internal multiples $[14,16]$. This method can be applied in a model-driven or a data-driven manner. However, these methods have common disadvantages. Firstly, the prediction-subtraction methods require complete and regular datasets. Thus, data interpolation processes should be implemented for irregular or incomplete data $[25,26]$ and the removal quality relies on the interpolation accuracy. Secondly, the adaptive subtraction may cause the loss or distortion of primaries when they are overlapped with multiples. To solve this problem, Liu et al. introduced statistically estimated inverse source wavelet to attenuate the multiples and also effectively preserve the amplitude and continuity of primary reflections [27].

Groenestijn and Verschuur proposed a direct inversion method to separate primaries and surface-related multiples, referred to as the Estimation of Primaries by Sparsity Inversion (EPSI) $[28,29]$. The objective function of the EPSI method is the sum of the L2norm of the estimation residual and the sparsity-constraint of the primary impulse responses. The estimation residuals are the difference between the observed seismic data and the calculated data obtained by the summation of the estimated primaries and multiples. The EPSI method directly estimates primaries and multiples by minimizing the sparsity-constrained objective function rather than the prediction-subtraction process in the SRME method. Therefore, the waveform distortion and energy loss caused by the adaptive subtraction in SRME is avoided. In addition, the EPSI method estimates the primaries and multiples from the full wave fields. The primaries can be obtained simultaneously from the primary and the multiple reflections in the data. Therefore, it has the effect of wave field reconstruction, which overcomes the problems of the irregular and missing data presented in SRME. The robust EPSI uses the L1-norm minimization to constrain the sparsity of the primary responses to improve the sparseness and the accuracy of the primary impulse response estimation [30]. The EPSI method was extended to a generalized version, called the generalized EPSI [31,32], which gradually eliminates surface-related and subsurface interface-related multiples from the surface to the subsurface. Song et al. demonstrated that the generalized ESPI is more effective in preserving the primary waveform for estimating internal multiples than the other two prediction-and-subtraction methods, namely the CFP-based method and the surface-data based method [33]. Even though the generalized EPSI performs well in multiple elimina- 
tions, the high computational cost restricts its use in industry and thus further optimization is necessary.

In this paper, we briefly review the generalized EPSI method and discuss its advantages and disadvantages. Based on the analysis on the generalized EPSI, we present an interface-controlled strategy and an approximate formula of the primary impulse responses to improve the efficiency and flexibility of the generalized EPSI method. A twolayered synthetic data test is implemented to analyze the features of the approximation in the modified method, and the benchmark data, Pluto1.5 data [34] test is implemented to verify the effectiveness and feasibility of the interface-controlled multiple elimination by sparsity inversion.

\section{Methodology}

\subsection{The generalized EPSI method}

In order to remove both surface and internal multiples, Ypma et al. proposed the generalized EPSI method, which is an extension of the original EPSI procedure (see in Appendix A) [31,32]. In the generalized EPSI method, the internal multiples are categorized according to their shallowest reflector. The internal multiples generated by the $n$th interface $M_{n}$ are given by

$$
P_{n-1}=P_{n}+M_{n}
$$

where $P_{n}$ represents the wavefields without the multiples generated by the interfaces $(0 \sim n)$. When $n=0$, then $P_{-1}=P$, which is consistent with the EPSI method. This algorithm starts from the estimation of multiples related to the first reflection interface $(n=0)$, i.e. the surface, and gradually goes down to the 1st, 2 nd and deeper reflectors. The expanded form of Eq. (2.1) is given as

$$
P_{n-1}=X_{n} S-\widehat{X}_{n} \delta X_{n}^{H} \widehat{P}_{n-1}^{\prime},
$$

where the superscript ${ }^{\prime H \prime}$ represents the conjugate transpose of a matrix, $X_{n}$ represents the primary impulse response related to $P_{n}, S$ is the source signature, $\widehat{X}_{n}$ extracts the primary impulse responses of $0 \sim n$th reflectors from $X_{n}, \delta X_{n}$ represents the primary impulse responses of the $n$th reflector, $\widehat{P}_{n-1}^{\prime}$ excludes the primaries of the $n$th reflector $\delta P_{n}$ from $\widehat{P}_{n-1}$, which can be expressed as

$$
\begin{aligned}
\widehat{P}_{n-1} & =\widehat{P}_{n-1}^{\prime}+\delta P_{n} \\
& =\widehat{P}_{n-1}^{\prime}+\delta X_{n} S .
\end{aligned}
$$

The primaries $P_{n}$ are characterized by $P_{n}=X_{n} S$ and the multiples are calculated by $M_{n}=\widehat{X}_{n} \delta X_{n}^{H} \widehat{P}_{n-1}^{\prime}$. In particular, when $n=0$ then $P_{-1}=\widehat{P}_{-1}=P$ and $\delta X_{0}=-R$ (surface reflectivity), $\widehat{X}_{0}=X_{0}$. Fig. 1 depicts several important physical quantities used in the generalized EPSI method. 


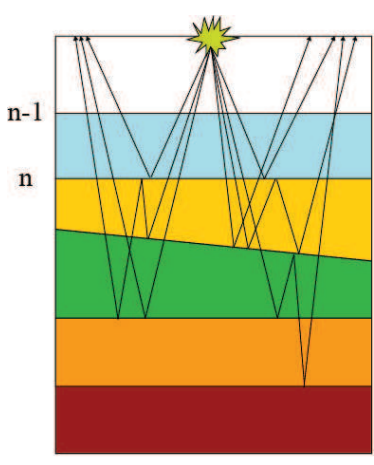

(a)

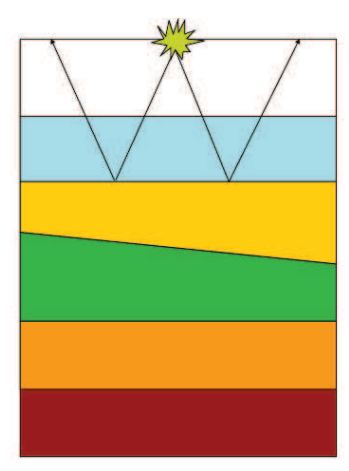

(b)

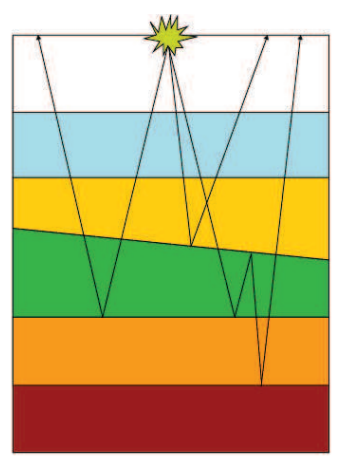

(c)

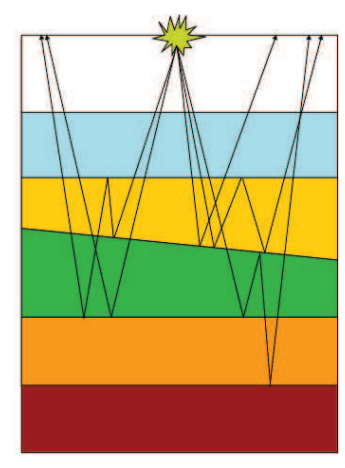

(d)

Figure 1: Important wavefields used in the generalized EPSI method [34]. (a) $\widehat{P}_{n-1}=\widehat{X}_{n-1} S$ extracts the primary responses of the 0 to $(n-1)$ th reflectors from $P_{n-1}$; (b) $\delta X_{n}$ represents the primary impulse responses of the $n$th reflector; (c) $\widehat{X}_{n}$ represents the impulse responses excluded from $X_{n}$ ( 0 to $n$th reflectors), including primaries and internal multiples that scatter downwards below the $n$th reflector; (d) $\widehat{P}_{n-1}^{\prime}$ excludes the primary responses of the $n$th reflector $\delta P_{n}$ from $\widehat{P}_{n-1}$, where $\delta P_{n}=\delta X_{n} S$.

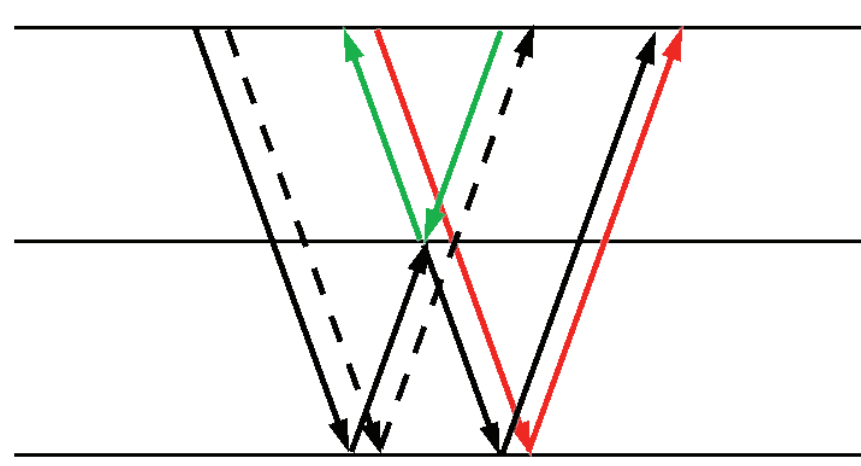

0

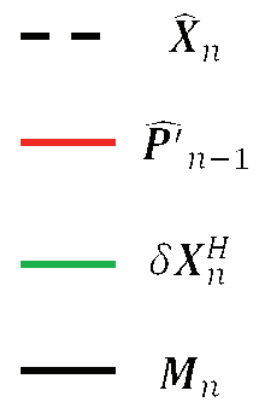

Figure 2: Schematic illustration of the formation of the internal multiples. The black-dotted, red, green, and black solid lines represent $\widehat{X}_{n}, \widehat{P}_{n-1}^{\prime}$, and $\delta X_{n}^{H}$, and $M_{n}$ (the internal multiples related to the $n$-th reflector), respectively.

The formation of the internal multiples is shown in Fig. 2 by depicting the wave path of various wavefields. The wave path of the $n$th reflector multiple has a "low-high-low" propagation pattern. Mathematically, and in practice, the $n$th reflector related multiples $M_{n}=\widehat{X}_{n} \delta X_{n}^{H} \widehat{P}_{n-1}^{\prime}$ are obtained through a multi-dimensional convolution and a multidimensional cross-correlation using three wavefield quantities.

As mentioned above, the generalized EPSI method eliminates the multiples from the surface to the subsurface. Removing the primary events (including the 0 to $n-1$ reflectors) from the left and right side of Eq. (2.2), we provide a fundamental equation that is 
used to construct the objective function of the generalized EPSI method:

$$
\widehat{P}_{n-1}=\left(\delta X_{n}+\widehat{X}_{n}\right) S-\widehat{X}_{n} \delta X_{n}^{H} \widehat{P}_{n-1}^{\prime} .
$$

According to Eq. (2.4), the objective function of the generalized EPSI method for estimating the $n$th reflector related multiples is given as below

$$
J_{n}=\sum_{\omega} \sum_{i r} \sum_{i s}\left\|\widehat{P}_{n-1}-\left(\delta X_{n}+\widehat{X}_{n}\right) S+\widehat{X}_{n} \delta X_{n}^{H} \widehat{P}_{n-1}^{\prime}\right\|_{2}^{2}+\left\|\widehat{X}_{n}\right\|_{0}+\left\|\delta X_{n}\right\|_{0} .
$$

The three unknown wavefields $\left(\widehat{X}_{n}, S\right.$ and $\left.\delta X_{n}\right)$ can be obtained by solving the objective function minimization problem in Eq. (2.5). Ypma et al. include a steepest-descent step to solve the minimization problem $[31,32]$

$$
\left\{\begin{array}{l}
\Delta \widehat{X}_{k}=\left(\widehat{P}_{n-1}-\left(\delta X_{n, i}+\widehat{X}_{n, i}\right) S_{i}+\widehat{X}_{n, i} \delta X_{n, i}^{H} \widehat{P}_{n-1}^{\prime}\right)\left(S_{i}^{H}-\widehat{P}_{n-1}^{\prime H} \delta X_{n, i}\right), \\
\widehat{X}_{n, i+1}=\widehat{X}_{n, i}+\alpha \Delta \underline{X}_{n}
\end{array}\right.
$$

where $\Delta$ represents the gradient direction, $\alpha$ is the scale factor, and the symbol ' $\quad$ ' represents the sparse constraint of a matrix or a vector. A more detailed theoretical derivation has been reported by Ypma et al. [31,32]. The multiples of the $n$th interface $M_{n}$ can be obtained by $M_{n}=-\widehat{X}_{n} \delta X_{n}^{H} \widehat{P}_{n-1}^{\prime}$ and are subtracted from the data after all the iterations. Alternatively, the subtraction process, can also be performed separately for each iteration by removing the multiples estimated during the current iteration.

\subsection{Interface-controlled strategy in multiple elimination by sparsity inversion}

As discussed above, the generalized EPSI method omits the adaptive subtraction step, so it can preserve the primaries better when they overlap with multiples, especially in shallow water explorations. In order to reduce the computational cost of the generalized EPSI, Ypma et al. introduced a macro-layer based scheme that divides the data into several groups of layers [31,32]. It was demonstrated that using a macro -layers based scheme does not have a significant effect on obtaining the good results. However, its high computational cost still remains the most challenging and significant problem. In this paper, we propose a practical strategy and an approximate formula, to further reduce the computation demands. We use a practical interface-controlled strategy that only estimates the multiples of specified interfaces, such as strong reflectors. In addition, we present an approximate formula of primary impulse responses that can decrease the computational costs.

In marine exploration, the primary imaging results are mostly contaminated by the reflectors that generate strong multiples, such as the sea surface, sea bottom, and subsurface salt dome structures. Although the generalized EPSI method can theoretically remove all types of multiples, its efficiency and flexibility still need to be improved. On 
the other hand, multiples related to comparatively weak interfaces are difficult to remove since in real data they are easily disturbed by other noises. Taking into consideration the fact that the internal multiples related to weak reflectors can be ignored in most cases, we can only estimate and remove the strong reflectors related multiples to improve the efficiency of the generalized EPSI method without significant quality reduction. Therefore, the primaries of the $n$ reflector in Eq. (2.1) can be rewritten as

$$
\begin{aligned}
P_{n} & =P-\sum_{n=1}^{N} M_{n} \\
& \approx P-\sum_{k=1}^{K} M_{k} \\
& =P-\sum_{k=1}^{K} \widehat{X}_{N(k)} \delta X_{N(k)}^{H} \widehat{P}_{n-1}^{\prime},
\end{aligned}
$$

where $K$ is the total number of chosen strong interfaces, $k=1,2, \cdots, K$ is the ordinal number of each chosen interface, and $N(k)$ represents the real order of the $k$ th specified reflector in the sequence of all $N$ reflectors. Based on Eq. (2.7), the objective function can be expressed as:

$$
\begin{aligned}
J_{N(k)}= & \sum_{\omega} \sum_{i r} \sum_{i s}\left\|\widehat{P}_{N(k)-1}-\left(\delta X_{N(k)}+\widehat{X}_{N(k)}\right) S+\widehat{X}_{N(k)} \delta X_{N(k)}^{H} \widehat{P}_{N(k-1)}^{\prime}\right\|_{2}^{2} \\
& +\left\|\widehat{X}_{N(k)}\right\|_{0}+\left\|\delta X_{N(k)}\right\|_{0}
\end{aligned}
$$

and further simplified as:

$$
J_{k}=\sum_{\omega} \sum_{i r} \sum_{i s}\left\|\widehat{P}_{k-1}-\left(\delta X_{k}+\widehat{X}_{k}\right) S+\widehat{X}_{k} \delta X_{k}^{H} \widehat{P}_{k-1}^{\prime}\right\|_{2}^{2}+\left\|\widehat{X}_{k}\right\|_{0}+\left\|\delta X_{k}\right\|_{0}
$$

The updated equations of the generalized EPSI method are still used to obtain the three unknowns $\widehat{X}_{k}, S$ and $\delta X_{k}$. However, in this version, only the primary impulse responses of the $k=1,2, \cdots, K$ th specified reflectors $\delta X_{k}$ are estimated.

The generalized EPSI method implements the sparse constraint based on two prerequisites. First, the primary impulse responses are band-limited spikes containing the wave path and the reflection information (i.e. the Green's function) and thus they have sparseness in temporal-spatial domain. In Practice, the generalized EPSI method implements the sparse constraint on $\widehat{X}_{k}$ by choosing a sample with maximum amplitude in the time window of the primary responses that obtained by the first equation in Eq. (2.6). During the picking process, it relies on the other prerequisite that the energy of the primaries is greater than that of the multiples. Thus, by the window picking, the focal energy near the source, the strong reflections above the $k$ th interfaces and the strong multiples are all excluded. The sparsification term $\delta X_{k}$ also requires a window picking that contains the $n$th reflector related primary impulse responses. Designing the window for picking the interface-related primary responses is very important. Fortunately, the temporal 
windows can be easily defined. For example, it is possible to roughly give a rectangle or hyperbolic window for each shot of collected data. In this manner, we can exclude the unrelated strong energies, such as the focused energy near the shot, artifacts above the first arrivals and the primaries of other strong reflectors. Next, the samples with the largest amplitude are selected as the center axis of the window. Finally, the rectangular or hyperbolic window is reduced or narrowed around the center point.

The source signature $S_{i+1}$ is estimated by least-square fitting $\delta X_{k, i+1}+\widehat{X}_{k, i+1}$ and $\widehat{P}_{k-1}^{\prime}+$ $\widehat{X}_{k, i+1} \delta X_{k, i+1}^{H} \widehat{P}_{k-1}^{\prime}$ in time domain. The iterations for the estimation of the $k$ th interface related multiples $M_{k}$ are continued until some criterion for convergence is met.

In the interface-controlled EPSI method, the computational time is approximately proportional to the number of involved reflectors. Compared with the $N$ times estimations of all the multiples performed in the generalized ESPI method in Eq. (2.5), the interface-controlled strategy solves the minimization problem only $K<N$ times. The determination of the number of specified reflectors depends on the balance between the calculation cost and removal quality. Usually, the energies of most internal multiples in the data are those related to a few strong reflectors. Therefore, using the interface-controlled strategy can remove most of the internal multiples in fewer computations than in the generalized EPSI. In the test of the benchmark Pluto data (see in Section 3.2), it provided fairly good results by only estimating and removing the surface related and the sea bottom related multiples.

\subsection{The approximate formula for estimating primary impulse responses}

In Eq. (2.6), the right-hand side of the first equation can be split into two parts as shown below:

$$
\begin{aligned}
\Delta \widehat{X}_{k}= & \left(\widehat{P}_{k-1}-\left(\delta X_{k, i}+\widehat{X}_{k, i}\right) S_{i}+\widehat{X}_{k, i} \delta X_{k, i}^{H} \widehat{P}_{k-1}^{\prime}\right) S_{i}^{H} \\
& -\left(\widehat{P}_{k-1}-\left(\delta X_{k, i}+\widehat{X}_{k, i}\right) S_{i}+\widehat{X}_{k, i} \delta X_{k, i}^{H} \widehat{P}_{k-1}^{\prime}\right) \widehat{P}_{k-1}^{\prime H} \delta X_{k, i} .
\end{aligned}
$$

The first part can be expressed as the contribution from the primaries themselves for estimating the primary impulse responses whilst the second part can be considered as the contribution from the internal multiples. It has been demonstrated that EPSI has a good performance in reconstructing missing data by estimating the primaries from the surface multiples [28]. Its success is attributed to the sufficiently strong energy of the surface multiples. Similarly, in the generalized EPSI method, the internal multiples are theoretically useful in estimating or reconstructing primaries. However, in many cases, it is difficult to reconstruct primaries from the usually weak internal multiples. On the other hand, it should be noted that in each iteration, the main computations use: the Fast Fourier Transform (FFT), the Inverse Fast Fourier Transform (IFFT) of several wavefields, the convolution for the internal multiples estimation, and the correlation for obtaining the second term in Eq. (2.10). Another reason why the generalized EPSI requires large numbers of computations is due to the correlation process between two wavefields. Taking the above into consideration, we deduce an approximation formula of Eq. (2.10) as 
shown below:

$$
\Delta \widehat{X}_{k}=\left(\widehat{P}_{k-1}-\left(\delta X_{k, i}+\widehat{X}_{k, i}\right) S_{i}+\widehat{X}_{k, i} \delta X_{k, i}^{H} \widehat{P}_{k-1}^{\prime}\right) S_{i}^{H} .
$$

Omitting the second term in Eq. (2.10) significantly reduces the computational cost of estimating the primary impulse responses. It should be noted that the approximations can be only used when internal multiples are weak. Fortunately, internal multiples are comparatively weak and thus the approximation can be applied in many cases. In order to improve the flexibility, one can selectively use the above approximate formula for removing weak interface related multiples whilst using the conversional formula in Eq. (2.6) to remove strong internal multiples.

The generalized EPSI method is a nested iterative algorithm, i.e. the outer loop relates to the interface and the inner loop relates to the estimation iterations of the current interface. The procedure for implementing the generalized EPSI method using the interface-controlled strategy is described as below:

1. Define the temporal window or boundaries of the specified interfaces that related to the primaries; initialize $\widehat{X}_{k}, S$ and $\delta X_{k}$;

2. Start the outer loop $k=1,2, \cdots, K$ to estimate the $k$ th related multiples $M_{k}$;

3. Calculate $\Delta \widehat{X}_{k}$, and then sparsify the result by picking samples in the time window with the maximum amplitude; update $\widehat{X}_{k, i}$;

4. In a Similar manner, obtain $\delta X_{k}$;

5. Calculate the $k$ th reflector related multiples $M_{k}$;

6. Using a temporal-spatial domain, $S_{k, i+1}$ is estimated by least-square fitting $\delta X_{k, i+1}+$ $\widehat{X}_{k, i+1}$ and $\widehat{P}_{k-1}^{\prime}+\widehat{X}_{k, i+1} \delta X_{k, i+1}^{H} \widehat{P}_{k-1}^{\prime}$;

7. Based on the maximum number of iterations or the threshold value of the residuals, decide whether the iteration for the $k$ th multiples estimation should be stopped. If the end conditions are met, go to step 7); otherwise, repeat steps 3)-6);

8. If $k+1<K$, return to step 2), start the iterative estimation for the $(k+1)$ th interface related multiples $M_{k+1}$; otherwise, terminate the outer loop.

\section{Numerical results}

\subsection{Two-layered model data}

Initially, the modified method was tested using a synthetic two-layered model. The model has two interfaces, the surface and a strong subsurface reflector. Accordingly, the synthetic data contains primaries, surface-related multiples and internal multiples related to the subsurface interface. In the test, we removed all aforementioned multiples 

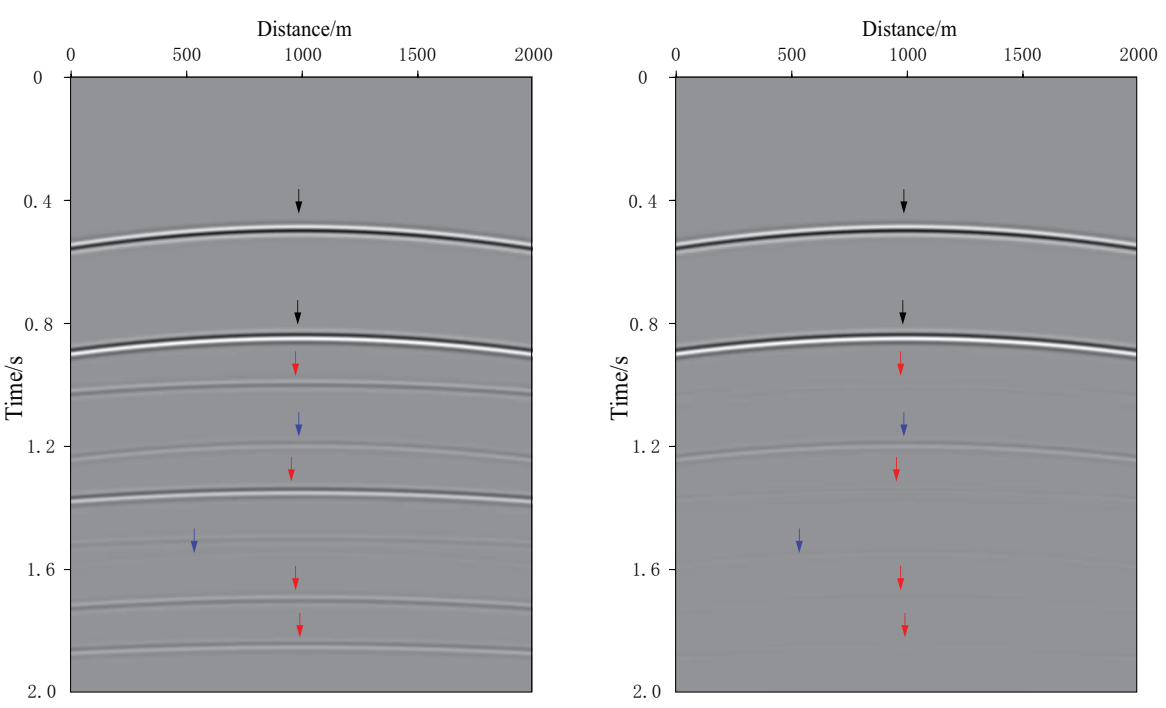

(a)
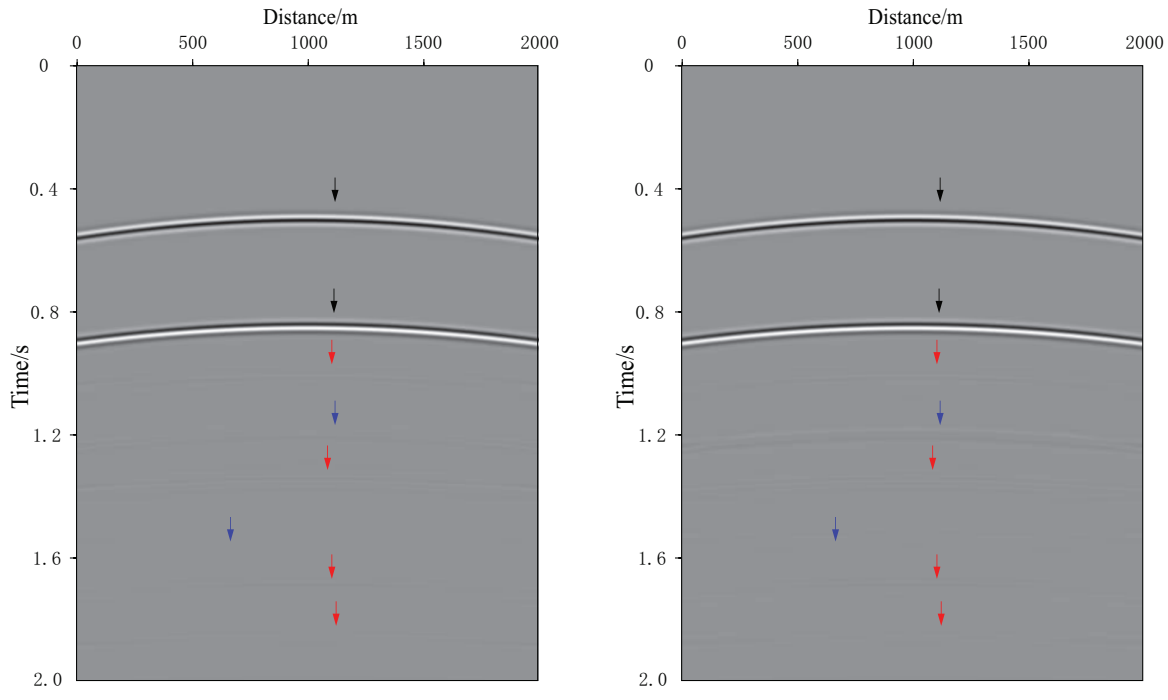

(c)

(d)

Figure 3: Comparison of the results of a two-layered synthetic data test using the original formula in Eq. (2.6) and the approximate formula in Eq. (2.11). (a) The original single shot record; (b) the results after surface multiples elimination; (c) the results after internal multiples removal by using the original formula of the generalized EPSI method in Eq. (2.6); (d) the results after internal multiples removal by using the approximate formula proposed in Eq. (2.11).

using the original formula in Eq. (2.6) and the proposed approximate formula in Eq. (2.11) respectively. Fig. 3a shows a raw common shot gather, where events of primaries are indicated by black arrows, with surface-related multiples by red arrows, and internal multiples by blue arrows. Fig. $3 \mathrm{~b}$ shows the results after surface multiples removal, which 


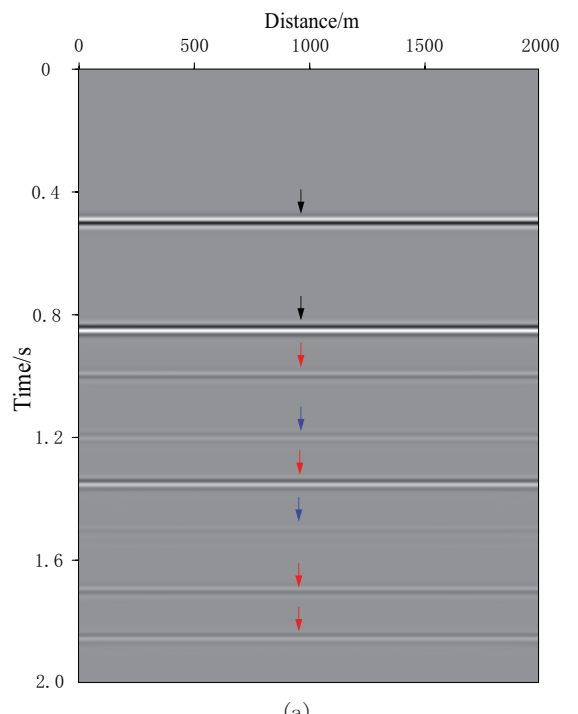

(a)

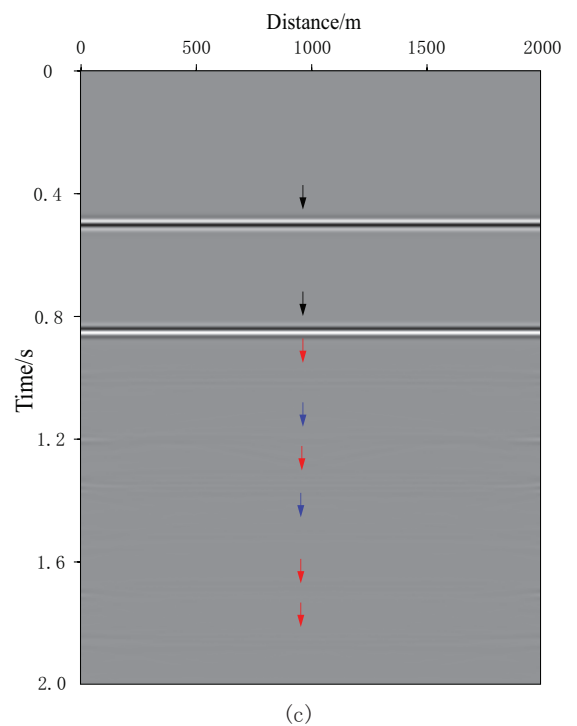

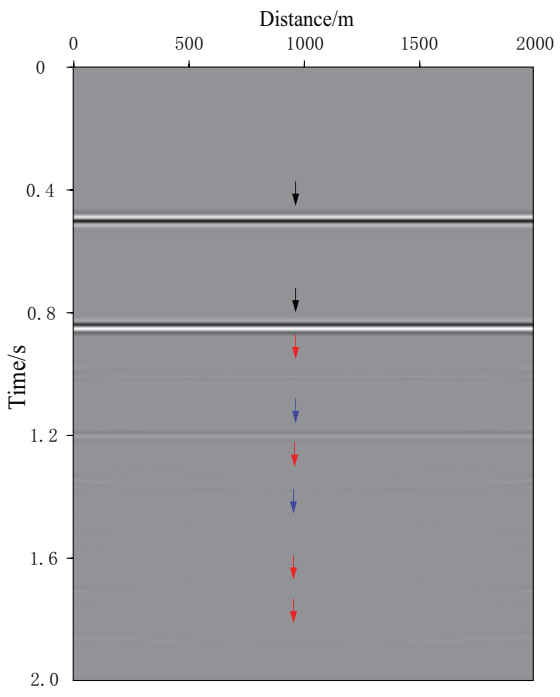

(b)

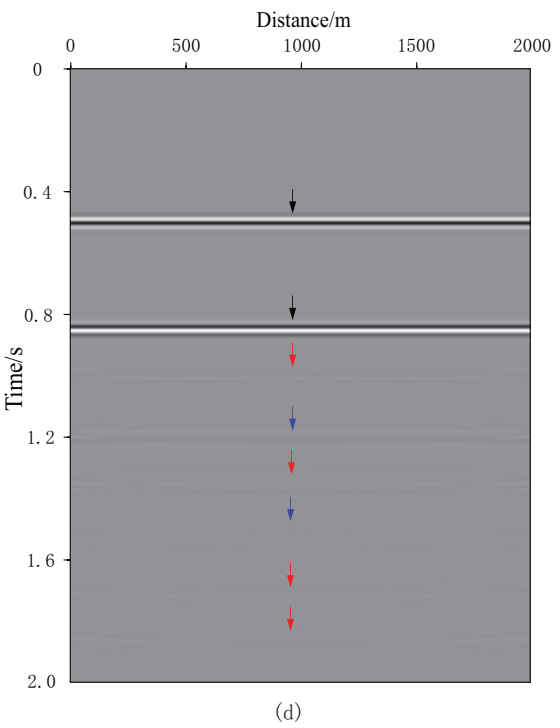

Figure 4: The zero-offset section of the results. (a) The zero-offset section of the original data; (b) the zerooffset section of the results after surface multiples removal; (c) the zero-offset section of the results after internal multiples removal by using the original formula of the generalized EPSI method in Eq. (2.6); (d) the zero-offset section of the results after internal multiples removal by using the approximate formula proposed in Eq. (2.11).

required 30 iterations. The surface multiples were well eliminated (comparing the events indicated by red arrows in Fig. 3a-b). Fig. 3c-d compares the results of removing internal multiples by using the conventional formula in Eq. (2.6) with the proposed approximate formula in Eq. (2.11). Fig. 4a-d show the zero-offset profiles of the raw data, the results after surface multiples elimination and after internal multiples elimination by using the 
original and the approximate calculation respectively. From these results, we demonstrate that the generalized EPSI method can successfully eliminate both surface-related and internal multiples. The approximation approach yields good results, removing most internal multiples (the maximum amplitude of the internal multiples is reduced by $90 \%$ ).

In the results of the approximation method, the internal multiples are not removed completely. There may be two main factors. Firstly, the interfaces in the model are designed with strong impedance difference so that the internal multiples are very strong. Secondly, the velocity model is very simple with horizontal reflecting layers. Therefore, the energy of primary responses estimated from the internal multiples may be concentrated better. Two aspects all affect the contributions from internal multiples on estimating primary responses. In fact, in real seismic data processing, most internal multiples are comparatively weak and the subsurface structure is very complex. In that case, the approximate formula is good enough to be used in the real data processing. It is well demonstrated in the benchmark test of the Pluto data in the next section.

\subsection{The Pluto1.5 synthetic data test}

The Pluto1.5 velocity model [34] contains seawater, several salt domes and large amounts of sedimentary layers, as shown in Fig. 5. The layers that generate the strongest multiples are the sea surface, the bottom of the seawater and the boundary of the salt domes. Therefore, using the interface-controlled strategy, we can lower the computational cost by only estimating and separating the surface related and the sea bottom related multiples. Additionally, the approximate formula in Eq. (2.11) is applied in the Pluto1.5 synthetic dataset test, removing the surface multiples and sea bottom related internal multiples.

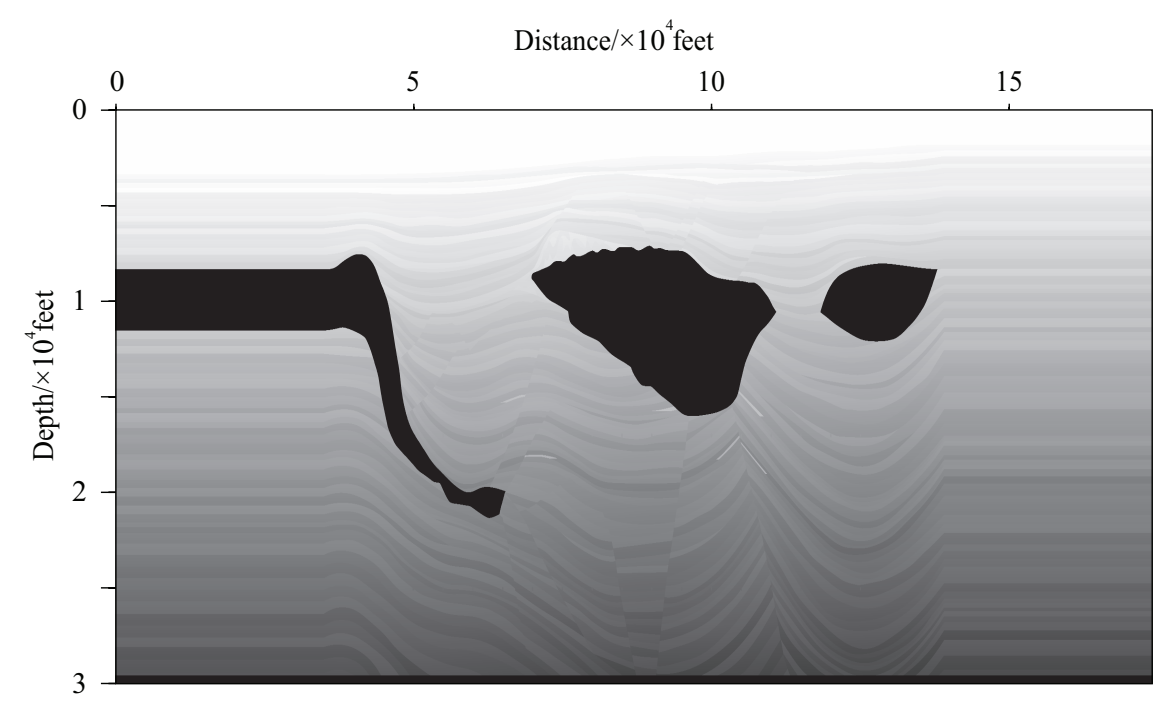

Figure 5: The Pluto velocity model [34]. 


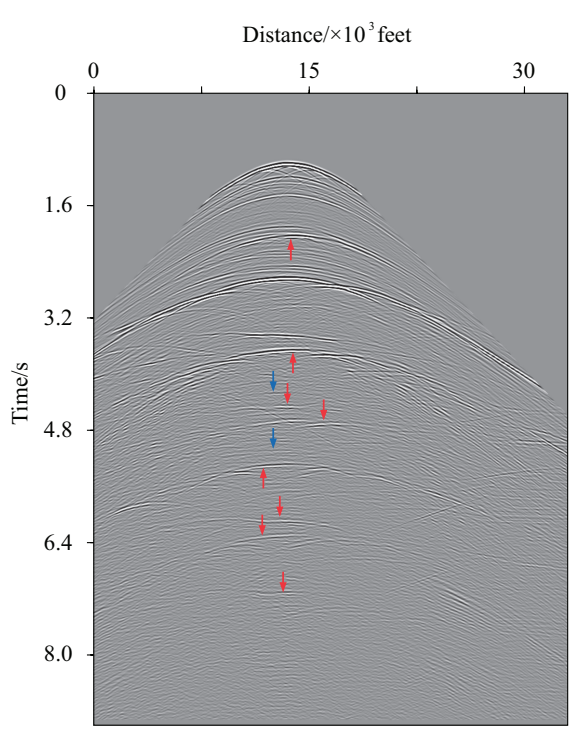

(a)

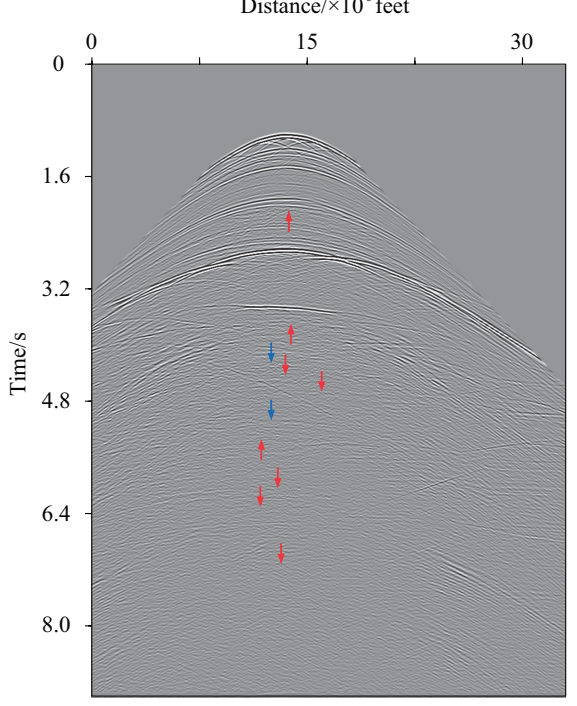

(c)

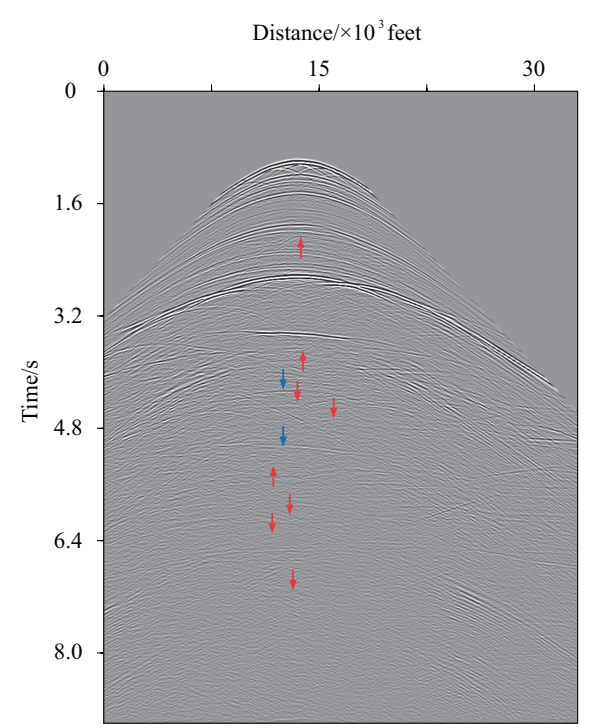

(b)

Distance $/ \times 10^{3}$ feet

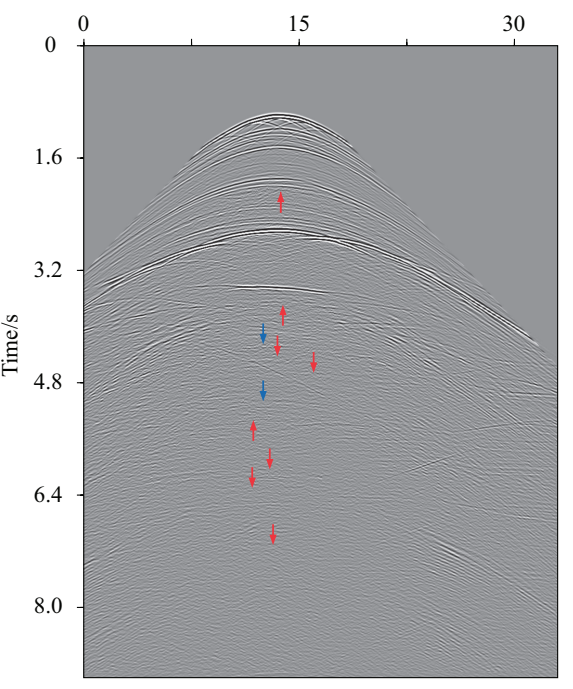

(d)

Figure 6: Pluto1.5 synthetic data test results. (a) The 580th shot record; (b) results after surface-related multiple removal; (c) results after seafloor-related internal multiples removal applying the original formula in Eq. (2.6); (d) results after seafloor-related internal multiples removal by the modified method.

Fig. 6a shows a common shot gather of the Pluto dataset. The red arrows indicate the surface-related multiples and the blue arrows indicate the sea bottom related internal multiples which arrive at $4 \mathrm{~s}$ to $5.6 \mathrm{~s}$. The shot record shows that the internal multiples generated by the strong interfaces are much weaker than the surface multiples. Fig. $6 \mathrm{~b}$ presents the results after the removal of surface-related multiples. As seen in Fig. 6b, 
Table 1: Comparison of the computational cost for estimating sea bottom related internal multiples when using the original formula in Eq. (2.6) and the approximate formula in Eq. (2.11).

\begin{tabular}{||c|c|c||}
\hline Computational parameters & The traditional formula & The approximate formula \\
\hline Total Iterations & 4 & 4 \\
\hline Computational nodes & $8 \mathrm{CPU}$ cores & $8 \mathrm{CPU}$ cores \\
\hline Memory & $18.345 \mathrm{G}$ & $9.785 \mathrm{G}$ \\
\hline Computational time & $2373.01 \mathrm{~s}$ & $747.751 \mathrm{~s}$ \\
\hline
\end{tabular}

after the removal of surface multiples, the internal multiples become sharper and easier to distinguish. It was found that the blue arrow pointed multiples, that related to the sea bottom, are the most prominent internal multiples. The result obtained using the traditional method and our interface-controlled strategy is shown in Fig. 6c-d respectively. Comparing Fig. 6a-c, we demonstrate that only estimating and removing the surface and the sea bottom related multiples gives satisfactory results, especially the surface multiples near $2 \mathrm{~s}, 3.8 \mathrm{~s}$ and $5.4 \mathrm{~s}$, and the internal multiples near 4-5.6s. The corresponding zero-offset sections of the results are shown in Fig. 7a-d. The procedure required a total of 30 iterations for the removal of surface multiples and only 4 iterations for the estimation of sea bottom related internal primaries. Table 1 shows a comparison between the computational demands for estimating sea bottom related internal multiples using the original formula in Eq. (2.6) and our modified version which uses an approximation Eq. (2.11). Under the same parallel computing environment, totally 4 iterations are required to predict the sea bottom related multiples using both the original formula and the approximate formula. The memory consumptions are 18.345G and 9.785G (only considering $3 \mathrm{D}$ variables and ignoring $1 \mathrm{D}$ or $2 \mathrm{D}$ variables) while the computational times are 2373.01s and 747.751s respectively. Note that the computations are counted only for estimation of sea bottom related internal multiples. It means that the approximation in Eq. (2.11) saves about $68 \%$ computations for estimating internal multiples.

\section{Conclusions}

We have proposed a modified method based on the generalized EPSI procedure to improve the computational efficiency in estimating primaries and internal multiples. We first introduced an interface-controlled strategy into the generalized EPSI method by only estimating multiples related to few strong reflection interfaces, such as the sea surface, sea bottom. The internal multiples related to weak reflectors have negligible effect on the imaging result. The interface-controlled strategy selectively separates multiples related to specified strong reflectors, according to the data characteristics, and thus significantly reduces the computational cost as there is no need to remove all multiples. The arithmetic operations and computational time were approximately proportional to the number of involved reflectors. The computational demands decreased linearly with a reduction in 


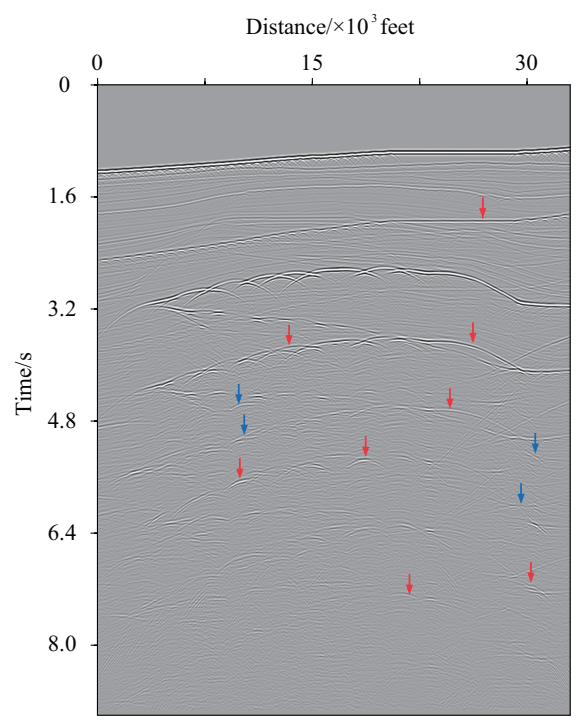

(a)

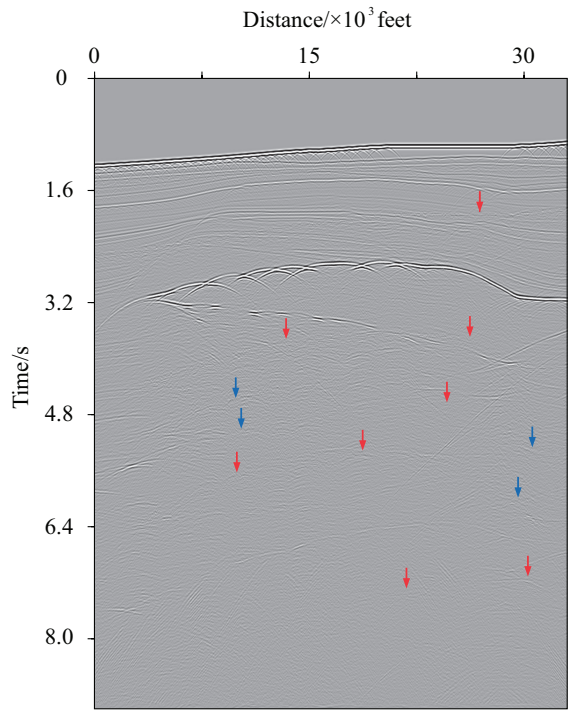

(c)

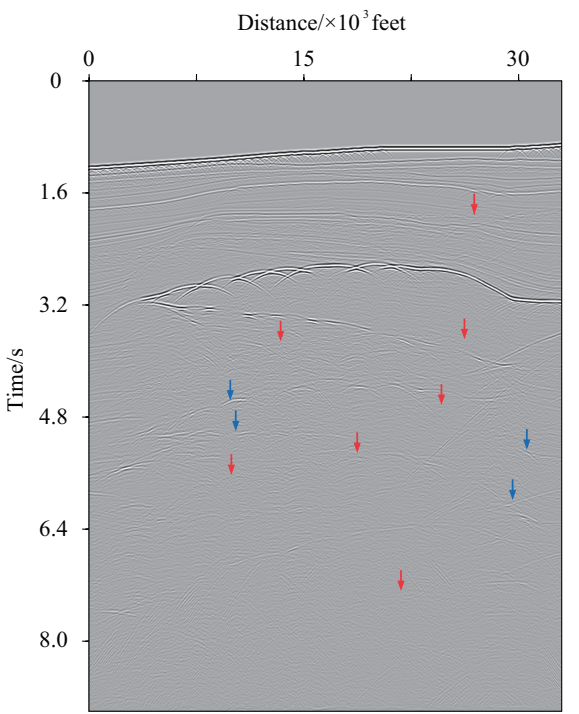

(b)

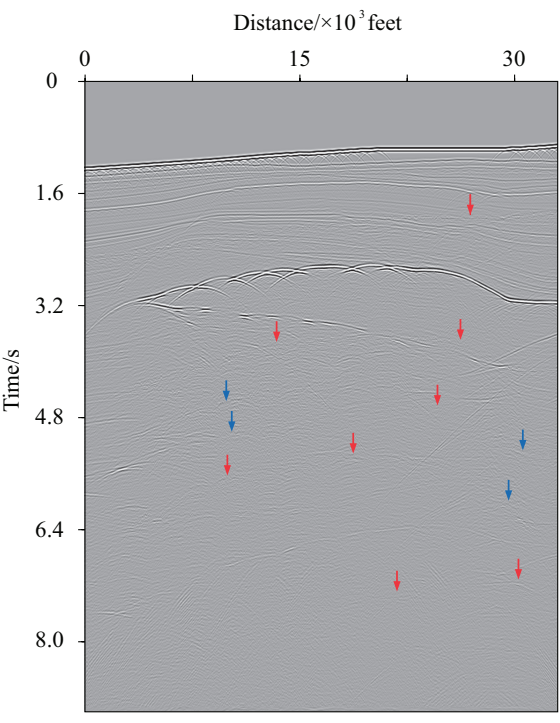

(d)

Figure 7: The zero-offset section of the results of the Pluto data test. (a) The zero-offset section of the original dataset; (b) the zero-offset section of the data after surface multiple removal; (c) the zero-offset section of the result after sea bottom related internal multiple removal by the original formula Eq. (2.6); (d) the zero-offset section of the data after sea bottom related internal multiple removal by our modified method.

the involved interfaces, whilst still obtaining satisfactory results. In addition, we proposed an approximate formula for estimating primary impulse responses by ignoring the contributions of internal multiples to the primary response estimation. Compared to other waves, the internal multiples are usually weak and thus have negligible effect on 
estimating the primary responses. We have applied this new method on the two-layered synthetic data and the Pluto1.5 benchmark data. In the Pluto dataset test, using the approximate formula reduces the computational time by about $68 \%$ for estimating the internal multiples related to the sea bottom. The results demonstrate the effectiveness and high-efficiency of the proposed method. The improvements in the modified method may increase its practicability of the generalized EPSI method in industry.

\section{Acknowledgments}

We extend our appreciation to two anonymous reviewers for valuable comments and advice regarding this work. We are especially grateful to Ru-Shan Wu for his continuous support to our research group. This study was supported by the National Natural Science Foundation of China (Grant Nos. 41704061 and 41730425), the National Major Project of China (Grant No. 2017ZX05008-007) and the Seismometry Talent Training Project of the China Earthquake Administration (Grant No. CEA-JC/QNCZ-18322).

\section{Appendix A}

Based on the iterative feedback model, the generalized EPSI method expresses the full wavefield including primaries and multiples in the frequency domain as below:

$$
P^{-}=P_{0}+M_{0}=X_{0} S^{+}+X_{0} R^{-} P^{-},
$$

where $P^{-}$represents the full wavefield, $P_{0}$ the primaries, $M_{0}$ the multiples, $X_{0}$ are the primary responses, $S$ is the seismic wavelet, $R^{-}$is the upgoing reflection coefficient of the free surface, superscript ${ }^{\prime-\prime}$ represents the upgoing wavefield, and subscript ${ }^{\prime}$ ' represents the depth of the surface $(n=0)$. In Eq. (A.1) the full wavefield $P^{-}$is the summation of the primaries $P_{0}$ and the multiples $M_{0}$. The term $P_{0}$ can be expressed by the multidimensional convolution of $X_{0}$ and $S$, where $S=s(\omega) I ; M_{0}$ can be obtained through multidimensional convolution of $X_{0}, R$ and $P^{-}$.

According to Eq. (A.1), the objective function of the EPSI method is established

$$
J=\sum_{\omega} \sum_{i s} \sum_{i r}\left\|P-X_{0} S-X_{0} R P\right\|_{2}^{2}+\left\|X_{0}\right\|_{0}
$$

where $\|\cdot\|_{0}$ represents "zero" norm, i.e. the number of non-zero elements. $\omega$, is and ir are the indexes related to the frequency, shot number and receiver number respectively. The symbol $\sum_{\omega} \sum_{i s} \sum_{i r}$. represents the summation of the objects related to all the frequencies, shots and receivers. In Eq. (A.2), the first term at the right-hand side of the equation is the total residual of the estimation of primaries and multiples, and the second term is the sparse constraint on primary impulse responses. This equation has two unknowns: the primary responses $X_{0}$ and the seismic wavelet $S^{+}$. The minimization problem of 
the objective function is solved based on the steepest descend algorithm. The difference between the EPSI method and the SRME method is that in the EPSI method the minimization of Eq. (A.2) ensures that the objective function goes to zero whilst in the SRME method it assumes that the primaries contain a minimum energy.

\section{References}

[1] Y. Liu, X. Chang, D. Jin, R. He, H. Sun, and Y. Zheng, Reverse time migration of multiples for subsalt imaging, Geophysics, 76(2011), 209-216.

[2] Y. Liu, H. Hu, X.B. Xie, Y. Zheng, and P. Li, Reverse time migration of internal multiples for subsalt imaging, Geophysics, 80(2015), S175-S185.

[3] Y. Liu, X. Liu, A. Osen, Y. Shao, H. Hu, and Y. Zheng, Least-squares time migration using controlled-order multiple reflections, Geophysics, 81(2016), S347-S357.

[4] N. Tu, A. Aravkin, T. Van Leeuwen, T. Lin, F. Herrmann, Source estimation with multiplesfast ambiguity-resolved seismic imaging, Geophys. J. Int., 205(2016): 1492-1511.

[5] N. Tu and F. Herrmann, Fast least-squares imaging with surface-related multiples: Application to a North Sea data set, The Leading Edge, 34(2015): 788-794.

[6] D. Hampson, Inverse velocity stacking for multiple elimination, J. Can. Soc. Expl. Geophys., 22(1986), 44-55.

[7] E. A. Robinson, Predictive decomposition of seismic traces, Geophysics, 22(1957), 767-778.

[8] M. T. Taner, Long period sea-floor multiples and their suppression, Geophysical Prospecting, 28(1980), 30-48.

[9] A. J. Berkhout and D. J. Verschuur, Estimation of multiple scattering by iterative inversion, Part I: Theoretical considerations, Geophysics, 62(1997), 1586-1595.

[10] D. J. Verschuur, Surface-related multiple elimination, an inversion approach, $\mathrm{PhD}$ Thesis, Delft University of Technology, 1991.

[11] D. J. Verschuur, A.J. Berkhout, and C.P.A. Wapenaar, Adaptive surface-related multiple elimination, Geophysics, 57(1992), 1166-1177.

[12] D. J. Verschuur, Estimation of multiple scattering by iterative inversion, Part II: Practical aspects and examples, Geophysics, 62(1997), 1596-1611.

[13] A. B. Weglein, F. A. Gasparotto, P. M. Carvalho, and R. H. Stolt, An inverse-scattering series method for attenuating multiples in seismic reflection data, Geophysics, 62(1997), 1975-1989.

[14] A. J. Berkhout and D. J. Verschuur, Removal of internal multiples with the common-focuspoint(CFP) approach: Part 1 - Explanation of the theory, Geophysics, 70(2005), V45-V60.

[15] H. Jacubowicz, Wave equation prediction and removal of interbed multiples. SEG Technical Programs Expanded Abstracts, 1998.

[16] D. J. Verschuur and A.J. Berkhout, Removal of internal multiples with the common-focuspoint CFP approach: Part 2 - Application strategies and data examples. Geophysics, 70(2005), V45-V60.

[17] J. R. Berryhill and Y. C. Kim, Deep-water peg legs and multiples: Emulation and suppression, Geophysics, 51(1986), 2177-2184.

[18] H. Hu, Y. Wang, X. Chang, and S. Xie, Migration of free-surface-related multiples: Removing artefacts using a water-layer model, Geophysics, 112(2015): 147-156.

[19] A. Pica and L. Delmas, Wave equation based internal multiple modelling in 3D, SEG Technical Program Expanded Abstracts,(2008), 2476-2480. 
[20] J. Wiggins, Attenuation of complex water-bottom multiples by wave-equation based prediction and subtraction, Geophysics, 53(1988), 1527-1539.

[21] S. E. Keydar, B. Landa, B. Gurevich, and B. Gelchinsky, Multiple reflection using wavefront characteristics of primary reflections, 59th Annual International Meeting, European Association of Geoscientists and Engineers, Expanded Abstracts, 1997.

[22] A. El-Emam, I. Moore, and A. Shabrawi, Interbed multiple prediction and attenuation: Case history from Kuwait. SEG Technical Program Expanded Abstracts, 24(2005), 448-451.

[23] J. Hembd, M. Griffiths, C.-O. Ting, and N. Chazalnoel, Application of interbed multiple attenuation in the Santos Basin, Brazil, SEG Technical Program Expanded Abstracts, 29(2010), 3451-3455.

[24] I. Moore, Practical implementation of interbed multiple attenuation, Australian Society of Exploration Geophysicists, 32(2001), 80-88.

[25] L. Bai, H. Lu, Y. Liu, and M. Khan, A fast joint seismic data reconstruction by sparsitypromoting inversion, Geophys. Prospect., 65(2017), 926-940.

[26] M. M. N. Kabir and D. J. Verschuur, Restoration of missing offsets by parabolic Radon transform, Geophys. Prospect., 43(1995), 347-368.

[27] Y. Liu, D. Jin, X. Chang, P. Li, H. Sun, and Y. Luo, Multiple subtraction using statistically estimated inverse wavelets, Geophysics, 75( 2010), WB247-WB254.

[28] G. J. V. Groenestijn and D. J. Verschuur, Estimating primaries by sparse inversion and application to near-offset data reconstruction, Geophysics, 74(2009), A23-A28.

[29] G. J. V. Groenestijn, Estimation of primaries and multiples by sparsity inversion. PhD Thesis, Delft University of Technology, 2010.

[30] T. T. Y. Lin and F.J. Herrmann, Robust estimation of primaries by sparse inversion via onenorm minimization. Geophysics, 78(2013), R133-R150.

[31] F. H. C. Ypma, Estimating primaries by sparsity Iinversion, a generalized approach, $\mathrm{PhD}$ Thesis. Delft University of Technology, 2010.

[32] F. H. C. F.H.C. and D.J. Verschuur, Estimating primaries by sparse inversion, a generalized approach, Geophys. Prospecti,, 61(2013), 94-108.

[33] J. Song, E. Verschuur, and X. Chen, Comparing three feedback internal multiple elimination methods, J. Appl. Geophys., 95(2013), 66-76.

[34] E. Bergsma, The Pluto1.5 2D synthetic dataset, Delft University of Technology, http://www.delphi.tudelft.nl/SMAART/pluto15.htm, 2000. 